\title{
Semiconductor polarization dynamics from the coherent to the incoherent regime: Theory and experiment
}

\author{
R. Lövenich, C. W. Lai, D. Hägele, and D. S. Chemla \\ Department of Physics, University of California at Berkeley and Materials Sciences Division, \\ Lawrence Berkeley National Laboratory, Berkeley, California 94720 \\ W. Schäfer \\ John von Neumann Institute for Computing, Forschungszentrum Jülich, 52425 Jülich, Germany
}

(Received 19 February 2002; published 10 July 2002)

\begin{abstract}
We explore the capability of a many-particle approach to the polarization dynamics of optically excited semiconductors. The theory contains the well-established theories for the coherent low excitation and incoherent high excitation regimes as limiting cases. The approach shows excellent agreement with four-wave mixing experiments where an incoherent background of carriers is created by a cw beam allowing to investigate the full range between the limiting cases.
\end{abstract}

DOI: 10.1103/PhysRevB.66.0453XX

PACS number(s): 78.47.+p, 78.66.Fd

\section{INTRODUCTION}

During the last decade investigations of the ultrafast optical response of semiconductors has brought us conceptual advances in our understanding of the dynamics of Coulomb correlations and electronic coherences in these materials. Indeed it is now possible to perform experiments with time resolution shorter than the scattering time scales of the fundamental elementary excitations and with interferometric control of the excitation density and energy. ${ }^{1,2}$ However, most of the work, experimental or theoretical, concentrates on either one of two completely different experimental situations, involving, respectively, coherently driven states ${ }^{3-7}$ or incoherently relaxing distributions. ${ }^{8-14}$ In real life this distinction is artificial since an initially coherently driven system naturally relaxes and eventually reaches thermodynamic equilibrium. When electron-hole pairs $(e-h)$ are excited by a short laser pulse they start oscillating coherently between the valence and conduction levels forming polarization waves whose quantum-mechanical phase is defined by that of the laser fields. This coherent regime lasts typically a few tens of femtoseconds (fs) and soon many-body interactions start between the polarization waves and the waves associated with the collective excitations of the sample, e.g., phonons, plasmons, etc. These interactions are not instantaneous; they are interferences with time scales defined by the period of the interacting waves. As they progress a number of processes begin to be "turned on." The lattice begins to react to the appearance of charges (phonons are absorbed or created) and the Coulomb potential starts to be screened (plasmons are absorbed or created). During this transient period incoherent populations are generated whose dynamics is still highly nonclassical. As scattering processes become effective, the coherence continues to decay, and is completely lost after many tens of femtoseconds. It is only then that the quasiparticles start to look more or less "classical." Clearly it is before the establishment of the classical regime that the most interesting physics occurs. In order to fix the ideas let us consider the case of the model semiconductor that we study in this paper: gallium arsenide (GaAs). In this material the LO-phonon period is $T_{L O}=115 \mathrm{fs}$, the period of a $\mathcal{N}_{e h}$
$=10^{17} \rightarrow 10^{18} \mathrm{~cm}^{-3}$ dense plasma ranges between $T_{P l}(\mathcal{N})$ $=100 \mathrm{fs}$ and $150 \mathrm{fs}$, and the "orbit" period of an exciton is $T_{X} \approx 1$ ps. All these times scales are well within range of modern experimental setups.

Up to very recently the theoretical approach to the physics of highly excited semiconductors was based on two complementary but distinct formalisms, the dynamically controlled truncation scheme (DCTS) and the real-time nonequilibrium Green's function (NGF) techniques. The DCTS formalism has been developed for describing the coherently driven $e-h$ pair system generated in the initial phase following laser excitation of the semiconductor in its ground state. ${ }^{15-20}$ The NGF approach is well suited for treating the effects of incoherent scattering and has been successfully applied to describe the non-Markovian behavior of relaxation processes. ${ }^{9,21-23}$

To obtain a thorough understanding of the evolution of $e-h$ pair systems from initial coherently driven states to relaxed ones it is important to have at hand a theoretical description that can bridge the two cases. Recently progress in that direction has been made at the level of the lowest $\left(\chi^{(3)}\right)$ nonlinearity. A many-particle theory has been developed, representing an approximation scheme that contains the NGF and the DCTS as limiting cases and provides a natural interpolation between these limits. ${ }^{18,24}$ It is based on a diagrammatic analysis of the interaction processes, which allows to renormalize up to infinite order in the Coulomb interaction the scattering between excitonic polarizations and the corresponding processes for the coherent contributions to the oneparticle distributions. In the coherent limit, perturbation development in terms of the applied field allows to relate the NGF to the DCTS and provides the basis for an ansatz used to reduce two-time Green's functions of the the NGF approach to single-time density matrices. This guarantees that the exact results of the DCTS are recovered in the coherent limit.

The approach was tested against experiment in the case of bulk three-dimensional (3D) GaAs in high magnetic field and it was found to describe well the transition from low to intermediate densities, i.e., for $\mathcal{N}_{e h}=1.5 \times 10^{15} \rightarrow 5$ 
$\times 10^{16} \mathrm{~cm}^{-3}$. Clearly, it is interesting to probe the range of applicability of the theory. This is the goal of this paper. We present a quantitative analysis of dephasing in quasi-2D GaAs quantum wells (QW's) where controlled amounts of incoherent carriers are introduced independently from the coherently driven $e-h$ pairs that participate to the phasesensitive four-wave mixing (FWM) experiments. ${ }^{25}$ We show that the theory can indeed describe the dephasing processes induced by carrier-carrier scattering and yet account for such important effects as exciton-biexciton quantum beats. The paper is organized as follows. In Sec. II we present the theory, and Sec. III describes the experimental setup and gives the main results, which are compared to numerical simulations and discussed in Sec. IV. Finally we conclude with a summary of recent progress and a discussion of future developments.

\section{THEORY}

As already mentioned our approach is a generalization of the second-order Born NGF theory, based on a diagrammatic analysis of the interaction processes. In this section we present the general framework and discuss how it includes the NGF and the DCTS as limiting cases. For completeness, a short derivation of the main theoretical steps is given in Appendix A whereas a longer derivation can be found in Refs. 18 and 24.

At low excitation densities and in the coherent limit the density matrix theory gives exact results for our manyparticle problem. Current NGF studies, however, are restricted to scattering processes high in the band-band continuum and at densities high enough that correlations can be neglected. In that case an expansion of the interaction processes in orders of the screened Coulomb interaction is justified. In practice these studies are usually limited to the second-order Born approximation.

The relevant microscopic quantity in both approaches is the transition amplitude

$$
P_{\mathbf{k}}^{e h}(t)=\left\langle\hat{e}_{\mathbf{k}}(t) \hat{h}_{-\mathbf{k}}(t)\right\rangle=i \hbar G_{e h \mathbf{k}}^{<}(t, t) .
$$

Within the Hartree-Fock approximation $P_{\mathbf{k}}^{e h}(t)$ satisfies the equation of motion:

$$
\begin{aligned}
{[-i \hbar} & \left.\frac{\partial}{\partial t}-\varepsilon_{\mathbf{k}}^{h}-\varepsilon_{\mathbf{k}}^{e}-i \hbar \gamma\right] P_{\mathbf{k}}^{e h} \\
= & \mathbf{d}_{e h} \mathbf{E}\left[1-f_{\mathbf{k}}^{e}-f_{\mathbf{k}}^{h}\right]-\sum_{\mathbf{k}^{\prime}} w_{\mathbf{k}^{\prime}} P_{\mathbf{k}^{\prime}}^{e h} \\
& -\sum_{\mathbf{k}^{\prime}} w_{\mathbf{k}-\mathbf{k}^{\prime}}\left[\left(f_{\mathbf{k}^{\prime}}^{e}+f_{\mathbf{k}^{\prime}}^{h}\right) P_{\mathbf{k}}^{e h}-\left(f_{\mathbf{k}}^{e}+f_{\mathbf{k}}^{h}\right) P_{\mathbf{k}^{\prime}}^{e h}\right] \\
& +\left.i \hbar \frac{\partial}{\partial t} P_{\mathbf{k}}^{e h}\right|_{\text {scatt }},
\end{aligned}
$$

where $f_{\mathbf{k}}^{e}$ and $f_{\mathbf{k}}^{h}$ are the one-particle distribution functions and $w_{\mathbf{k}}$ is the screened Coulomb potential.

If the scattering contribution, i.e., the last term, and screening are neglected, Eq. (2), together with the corre- sponding ones for $f_{\mathbf{k}}^{e}$ and $f_{\mathbf{k}}^{h}$ form the well-known semiconductor Bloch equations. ${ }^{26}$ Therefore, to go beyond this level of description, the task is to find suited approximations for the scattering contribution.

If we want to expand the NGF theory to low densities, we must identify those diagrams in the second-order Born approximation that correspond to the expansion of the biexcitonic correlation to second order in the Coulomb interaction. These diagrams describe polarization scattering. Those diagrams have to be dressed by higher-order Coulomb interactions which describe the interactions between two electrons and two holes. The only restriction we impose on the additional Coulomb interaction lines is that they are ladderlike for a given pair of particles. This is exactly the same level of approximation used to describe the formation of excitons. The ladder approximation allows us to derive a recursion formula relating the $n$ th- and $(n-1)$ th-order contributions to the exciton-exciton interaction. This recursion formula can be mapped onto an equation of motion for the biexcitonic correlation.

Within this general strategy two important issues must be considered: (i) identifying the diagrams that must be renormalized and performing this renormalization, and (ii) finding a consistent approximation for making the NGF problem solvable. In the context of (i) we note that beside the polarization scattering diagrams another class of diagrams must be renormalized. The one-particle distribution functions can be split into a coherent and an incoherent part,

$$
f_{\mathbf{k}}^{e}=f_{\mathbf{k}}^{e \text { coh }}+f_{\mathbf{k}}^{e \text { inc }} .
$$

Here we assume that the incoherent contribution is dominated by free carriers and adequately described within the second-order Born approximation. Higher-order interactions giving rise to the formation of biexcitons are taken into account for the coherent contributions only. The coherent part is determined by the product of two polarizations, i.e.,

$$
f_{\mathbf{k}}^{e c o h}=\sum_{h} P_{k}^{e h} P_{k}^{h e}
$$

and it has to be renormalized because it also gives rise to exciton-exciton interactions. As for (ii), it is necessary to find a consistent approximation for reducing the two-time Green's functions to single-time functions. Even at the Hartree-Fock approximation level, treating nonequilibrium Green's functions in a two-time basis is a difficult task ${ }^{17}$ and is not feasible at the level of four-particle correlations which we want to include. Furthermore, the standard approximation to achieve this goal, i.e., the generalized Kadanoff-Baym ansatz, ${ }^{27}$ does not reproduce the density matrix theory results in the low excitation regime. Therefore, we use another ansatz which agrees with the known exact results in the low excitation densities. Our procedure is justified by solving the Dyson equation linear in the external field. The details of our derivation are given in Appendix A. A quasiparticle approximation for the spectral functions in Eqs. (A3) and (A5) gives for the off-diagonal elements of the Green's functions in the leading order 
$G_{c v \mathbf{k}}^{<}\left(t, t^{\prime}\right)=G_{c v \mathbf{k}}^{<}(t, t) e^{i \varepsilon_{\mathbf{k}}^{v}\left(t-t^{\prime}\right)}=-\frac{i}{\hbar} P_{\mathbf{k}}^{c v}(t) e^{i \varepsilon_{\mathbf{k}}^{v}\left(t-t^{\prime}\right)}$

and

$G_{c v \mathbf{k}}^{>}\left(t, t^{\prime}\right)=G_{c v \mathbf{k}}^{>}\left(t^{\prime}, t^{\prime}\right) e^{i \varepsilon_{\mathbf{k}}^{c}\left(t-t^{\prime}\right)}=-\frac{i}{\hbar} P_{\mathbf{k}}^{c v}\left(t^{\prime}\right) e^{i \varepsilon_{\mathbf{k}}^{c}\left(t-t^{\prime}\right)}$.

Similarly we obtain for the coherent part of the diagonal Green's functions

$$
G_{c c \mathbf{k}}^{<\operatorname{coh}}\left(t, t^{\prime}\right)=e^{i \varepsilon_{\mathbf{k}}^{c}\left(t-t^{\prime}\right)} \sum_{v} G_{c v \mathbf{k}}^{<}(t, t) G_{v c \mathbf{k}}^{<}\left(t^{\prime}, t^{\prime}\right)
$$

$$
=-\frac{1}{\hbar^{2}} e^{i \varepsilon_{\mathbf{k}}^{c}\left(t-t^{\prime}\right)} \sum_{v} P_{\mathbf{k}}^{c v}(t) P_{\mathbf{k}}^{v c}\left(t^{\prime}\right) .
$$

Therefore, our approach complements the well-known Hartree-Fock term in Eq. (2) by a scattering term of the form

$$
\begin{aligned}
\left.i \hbar \frac{\partial}{\partial t} P_{\mathbf{k}}^{e h}\right|_{\text {scatt }}= & \sum_{\mathbf{k}^{\prime} \mathbf{q}, a=e, h} \sum_{b=\{e, h\}}\left[w_{\mathbf{q}}^{2}-\delta_{a, b} w_{\mathbf{q}} w_{\mathbf{k}-\mathbf{k}^{\prime}}\right] \times\left\{\left\{g ( \varepsilon _ { \mathbf { k } } ^ { a } - \varepsilon _ { \mathbf { k } + \mathbf { q } } ^ { a } + \varepsilon _ { \mathbf { k } ^ { \prime } + \mathbf { q } } ^ { b } - \varepsilon _ { \mathbf { k } ^ { \prime } } ^ { b } ) P _ { \mathbf { k } } ^ { e h } \left[\left[\left(1-f_{\mathbf{k}+\mathbf{q}}^{a}\right) f_{\mathbf{k}^{\prime}+\mathbf{q}}^{b}\left(1-f_{\mathbf{k}^{\prime}}^{b}\right)\right]\right.\right.\right. \\
& \left.\left.\left.+[f \rightleftharpoons(1-f)]-f_{\mathbf{k}^{\prime}+\mathbf{q}}^{b c 0 h}\right]\right\}-\left\{\mathbf{k} \rightleftharpoons(\mathbf{k}+\mathbf{q}), \mathbf{k}^{\prime} \rightleftharpoons\left(\mathbf{k}^{\prime}+\mathbf{q}\right)\right\}\right\}-\sum_{\mathbf{k}^{\prime} \mathbf{q} ; a, b=e, h}\left(1-\delta_{a, b}\right) w_{\mathbf{q}} w_{\mathbf{k}-\mathbf{k}^{\prime}} \\
& \times g\left(\varepsilon_{\mathbf{k}+\mathbf{q}}^{a}-\varepsilon_{\mathbf{k}}^{a}+\varepsilon_{\mathbf{k}^{\prime}}^{b}-\varepsilon_{\mathbf{k}^{\prime}+\mathbf{q}}^{b}\right)\left\{\left\{P_{\mathbf{k}+\mathbf{q}}^{e h}\left[\left[\left(1-f_{\mathbf{k}}^{a}\right) f_{\mathbf{k}^{\prime}+\mathbf{q}}^{b}\left(1-f_{\mathbf{k}^{\prime}}^{a}\right)\right]+[f \rightleftharpoons(1-f)]-f_{\mathbf{k}^{\prime}+\mathbf{q}}^{b c o h}\right]\right\}-\left\{(\mathbf{k}+\mathbf{q}) \rightleftharpoons\left(\mathbf{k}^{\prime}+\mathbf{q}\right)\right\}\right\} \\
& +\sum_{\mathbf{k q} ; e^{\prime} h^{\prime}} w_{\mathbf{q}}\left[P_{\mathbf{k}^{\prime}}^{e^{\prime} h^{\prime}}-P_{\mathbf{k}^{\prime}+\mathbf{q}}^{e^{\prime} h^{\prime}}\right]^{*} \times\left[B_{\mathbf{k}+\mathbf{q}, \mathbf{k}, \mathbf{k}^{\prime}, \mathbf{k}^{\prime}+\mathbf{q}}^{e h h^{\prime} h^{\prime}}+B_{\mathbf{k}, \mathbf{k}+\mathbf{q}, \mathbf{k}^{\prime}+\mathbf{q}, \mathbf{k}^{\prime}}^{e h}\right]
\end{aligned}
$$

where $g(\omega)=P(1 / \omega)+i \pi \delta(\omega)$ and the $B_{\mathbf{k}+\mathbf{q}, \mathbf{k}, \mathbf{k}^{\prime}, \mathbf{k}^{\prime}+\mathbf{q}}^{e h e^{\prime}}$ are the biexcitonic correlation functions.

The first term of Eq. (8) is the second-order contribution to scattering, which we write in the Markovian limit. Compared to the usual NGF results the coherent part of the oneparticle distributions is substracted, leaving in the linear density regime only the incoherent part. The coherent contribution is part of the second term which couples the biexcitonic correlation functions to the transition amplitude. It is worth noting the Markovian coupling of $P^{e^{\prime} h^{\prime *}}$ whereas the coupling of $B^{e h e^{\prime} h^{\prime}}$ is non-Markovian. This result, well known from density-matrix theory, is a consequence of our ansatz Eqs. (6) and (7) and could not be achieved by a generalized Kadanoff-Baym ansatz. We recover the coherent limit of the DCTS theory by assuming negligible screening and dephasing, which is valid in the low excitation regime. In this case the first term describing incoherent scattering vanishes and only the contribution of the biexcitonic corre- lation with the unscreened Coulomb interaction $w_{\mathbf{q}} \rightarrow v_{\mathbf{q}}$ is left over. The high-density limit is recovered as well. With increasing density the biexcitonic correlations die out due to scattering with free carriers. This allows a pertubative treatment of the Coulomb interaction. Using the Markov approximation and treating the biexcitonic correlations in secondorder Born approximation, the contributions of the coherent densities cancel out and we find the usual NGF result.

At intermediate densities the theory is not fully consistent and can be improved. The Markov approximation for the scattering with free carriers could be avoided. More important we treat the two-time functions by a low-density ansatz. However at higher densities the influence of biexcitonic correlations decreases, so that this approximation should have only minor consequences. Since our approach includes the two limiting cases exactly, we expect that it gives reasonable results at intermediate densities.

The set of equations of motion is completed by the equation for the biexcitonic correlation:

$$
\begin{aligned}
& {\left[i \hbar \frac{\partial}{\partial t}+\varepsilon_{\mathbf{k}+\mathbf{q}}^{e}+\varepsilon_{\mathbf{k}}^{h}+\varepsilon_{\mathbf{k}^{\prime}}^{e^{\prime}}+\varepsilon_{\mathbf{k}^{\prime}+\mathbf{q}}^{h^{\prime}}+\sigma_{\mathbf{k}+\mathbf{q}}^{e}+\sigma_{\mathbf{k}}^{h}+\sigma_{\mathbf{k}^{\prime}}^{e^{\prime}}+\sigma_{\mathbf{k}^{\prime}+\mathbf{q}}^{h^{\prime}}\right] B_{\mathbf{k}+\mathbf{q}, \mathbf{k}, \mathbf{k}^{\prime}, \mathbf{k}^{\prime}+\mathbf{q}}^{e h e^{\prime}}+\sum_{\mathbf{q}^{\prime}}\left[\left(\sigma_{\mathbf{k}+\mathbf{q}, \mathbf{q}^{\prime}}^{e}+\sigma_{\mathbf{k}, \mathbf{q}^{\prime}}^{h}\right) B_{\mathbf{k}+\mathbf{q}^{+}+\mathbf{q}^{\prime}, \mathbf{k}+\mathbf{q}^{\prime}, \mathbf{k}^{\prime}, \mathbf{k}^{\prime}+\mathbf{q}}^{e h e^{\prime}}\right.} \\
& \left.+\left(\sigma_{\mathbf{k}^{\prime}, \mathbf{q}^{\prime}}^{e^{\prime}}+\sigma_{\mathbf{k}^{\prime}+\mathbf{q}, \mathbf{q}^{\prime}}^{h^{\prime}}\right) B_{\mathbf{k}+\mathbf{q}, \mathbf{k}, \mathbf{k}^{\prime}+\mathbf{q}^{\prime}, \mathbf{k}^{\prime}+\mathbf{q}^{+}+\mathbf{q}^{\prime}}^{e h h^{\prime} h^{\prime}}\right]+\sum_{\mathbf{q}^{\prime}} w_{\mathbf{q}^{\prime}}\left[B_{\mathbf{k}+\mathbf{q}^{+} \mathbf{q}^{\prime}, \mathbf{k}, \mathbf{k}^{\prime}-\mathbf{q}^{\prime}, \mathbf{k}^{\prime}+\mathbf{q}^{\prime}}^{e h h^{\prime}}+B_{\mathbf{k}+\mathbf{q}, \mathbf{k}+\mathbf{q}^{\prime}, \mathbf{k}^{\prime}, \mathbf{k}^{\prime}+\mathbf{q}^{\prime}+\mathbf{q}^{\prime}}^{e h h^{\prime}}\right. \\
& \left.-B_{\mathbf{k}+\mathbf{q}^{+} \mathbf{q}^{\prime}, \mathbf{k}+\mathbf{q}^{\prime}, \mathbf{k}^{\prime}, \mathbf{k}^{\prime}+\mathbf{q}}^{e h{ }^{\prime}}-B_{\mathbf{k}+\mathbf{q}, \mathbf{k}, \mathbf{k}^{\prime}+\mathbf{q}^{\prime}, \mathbf{k}^{\prime}+\mathbf{q}^{\prime}+\mathbf{q}^{\prime}}^{e h h^{\prime} h^{\prime}}-B_{\mathbf{k}+\mathbf{q}^{+} \mathbf{q}^{\prime}, \mathbf{k}, \mathbf{k}^{\prime}, \mathbf{k}^{\prime}+\mathbf{q}^{\prime}+\mathbf{q}^{\prime}}^{e}-B_{\mathbf{k}+\mathbf{q}, \mathbf{k}+\mathbf{q}^{\prime}, \mathbf{k}^{\prime}+\mathbf{q}^{\prime}, \mathbf{k}^{\prime}+\mathbf{q}}^{e h h^{\prime}}\right] \\
& =w_{\mathbf{q}}\left(P_{\mathbf{k}}^{e h}-P_{\mathbf{k}+\mathbf{q}}^{e h}\right)\left(P_{\mathbf{k}^{\prime}}^{e^{\prime} h^{\prime}}-P_{\mathbf{k}^{\prime}+\mathbf{q}}^{e^{\prime} h^{\prime}}\right)-w_{\mathbf{k}-\mathbf{k}^{\prime}}\left(P_{\mathbf{k}}^{e^{\prime} h}-P_{\mathbf{k}^{\prime}}^{e^{\prime} h}\right)\left(P_{\mathbf{k}^{\prime}+\mathbf{q}}^{e h^{\prime}}-P_{\mathbf{k}+\mathbf{q}}^{e h^{\prime}}\right) \text {. }
\end{aligned}
$$


Here the diagonal parts of the one-particle self-energies are defined by

$$
\begin{aligned}
\sigma_{\mathbf{k}}^{a}= & \sum_{\mathbf{q}^{\prime} \mathbf{k}^{\prime} ; b=\{e, h\}}\left[w_{\mathbf{q}}^{2}-\delta_{a, b} w_{\mathbf{q}^{\prime}} w_{\mathbf{k}-\mathbf{k}^{\prime}}\right] \\
& \times g\left(\varepsilon_{\mathbf{k}}^{a}-\varepsilon_{\mathbf{k}+\mathbf{q}^{\prime}}^{a}+\varepsilon_{\mathbf{k}^{\prime}+\mathbf{q}^{\prime}}^{b} \varepsilon_{\mathbf{k}^{\prime}}^{b}\right) \times\left[\left[\left(1-f_{\mathbf{k}+\mathbf{q}^{\prime}}^{a}\right)\right.\right. \\
& \left.\left.\times f_{\mathbf{k}^{\prime}+\mathbf{q}^{\prime}}^{b}\left(1-f_{\mathbf{k}^{\prime}}^{b}\right)\right]+[f \rightleftharpoons(1-f)]\right],
\end{aligned}
$$

and the off-diagonal parts as

$$
\begin{aligned}
\sigma_{\mathbf{k}, \mathbf{q}^{\prime}}^{a}= & \sum_{\mathbf{k}^{\prime} ; b=\{e, h\}}\left[w_{\mathbf{q}}^{2}-\delta_{a, b} w_{\mathbf{q}} w_{\mathbf{k}-\mathbf{k}^{\prime}}\right] \\
& \times g\left(\varepsilon_{\mathbf{k}+\mathbf{q}^{\prime}}^{a}-\varepsilon_{\mathbf{k}}^{a}+\varepsilon_{\mathbf{k}^{\prime}}^{b}-\varepsilon_{\mathbf{k}^{\prime}+\mathbf{q}^{\prime}}^{b}\right) \times\left[\left[\left(1-f_{\mathbf{k}}^{a}\right)\right.\right. \\
& \left.\left.\times f_{\mathbf{k}^{\prime}}^{b}\left(1-f_{\mathbf{k}^{\prime}+\mathbf{q}^{\prime}}^{b}\right)\right]+[f \rightleftharpoons(1-f)]\right] .
\end{aligned}
$$

These terms give rise to an energy shift of the biexcitonic spectrum, but even more important, they describe the dephasing of the biexcitonic correlation functions under the influence of free carriers. Aside from these self-energy terms we recover the DCTS equation of motion.

\section{EXPERIMENT}

The method of choice for testing the approach is timeintegrated four-wave mixing (TI-FWM), since it probes both carrier correlation and coherence. As a key experiment we perform TI-FWM on a QW sample that already contains an incoherent background of electrons and holes that is continously created by a continuous wave (cw) laser. This situation displays an important test for the approach, because the DCTS cannot treat an incoherent background carrier distribution and the NGF is only valid in the high excitation limit. The full capability of the approach is demonstrated by choosing an excitation regime high enough to exhibit four-particle Coulomb correlation (in particular biexcitonic) signatures in the TI-FWM, but yet well below the plasma regime where the Coulomb potential is strongly screened. We determine this regime through two preliminary experiments, (i) an excitation-dependent pulse transmission of short laser pulses and (ii) TI-FWM without an incoherent background of carriers.

The sample under investigation is an undoped multiple GaAs/(AlGa)As QW grown by molecular beam epitaxy. The active region contains 10 periods of $14-\mathrm{nm}$ GaAs layers separated by $10-\mathrm{nm} \mathrm{Al}_{0.3} \mathrm{Ga}_{0.7} \mathrm{As}$ barriers. The $\mathrm{QW}$ structure is sandwiched between 500-nm and $490-\mathrm{nm} \mathrm{Al}_{0.3} \mathrm{Ga}_{0.7} \mathrm{As}$ layers, and capped with a 10-nm GaAs layer to avoid oxidation. The sample substrate is removed by wet etching to allow for transmission experiments, both sides are antireflection coated with $\mathrm{Sc}_{2} \mathrm{O}_{3}$ and the sample is glued on a $c$-axis sapphire disk.

We use a mode-locked Ti:sapphire laser that emits 100200 -fs pulses at a repetition rate of $100 \mathrm{MHz}$ with an average power of $300 \mathrm{~mW}$. Another Ti:sapphire laser is run in the $\mathrm{cW}$ mode for creating an incoherent background of carriers in the sample. The diameters of the focused laser spot on the

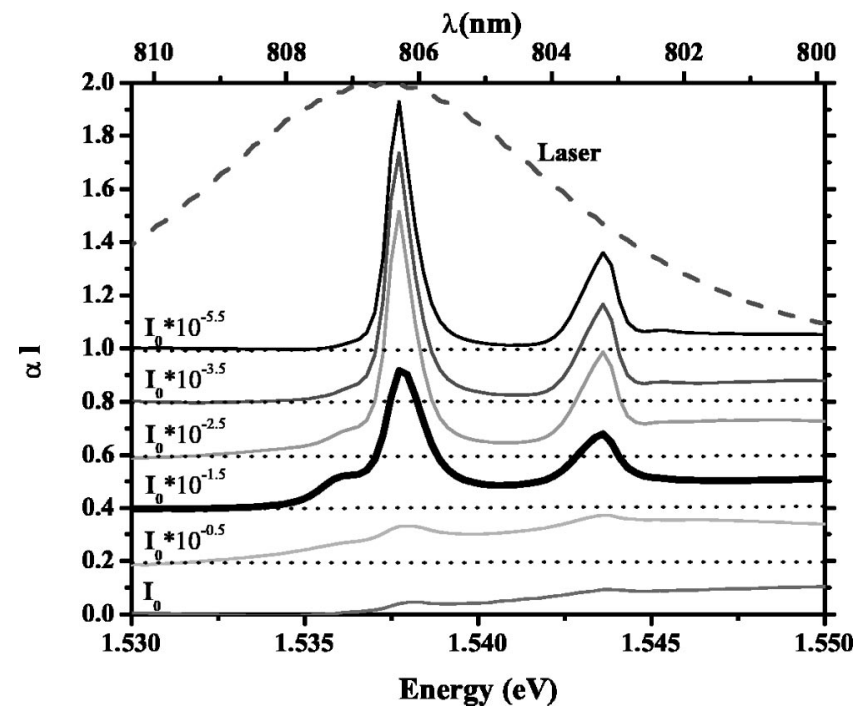

FIG. 1. Excitation intensity dependence of the absorption coefficient spectra of the sample. The curves are displaced by 0.2 consecutively from bottom to top for clarity. The dashed line is the spectrum of the laser pulse used to perform measurements. The intensity $I_{0}$ is about $10^{4} \mathrm{~W} \mathrm{~cm}^{-2}$.

sample of mode-locked and cw lasers are $27 \mu \mathrm{m}$, and $44 \mu \mathrm{m}$ respectively. The sample is always kept at a temperature below $5 \mathrm{~K}$ to minimize phonon effects.

We perform first transmission experiments with excitation intensities $10^{-5} I_{0} \rightarrow I_{0}$, where $I_{0} \approx 10 \mathrm{~kW} \mathrm{~cm}{ }^{-2}$. Absorption spectra, $\alpha(\omega)$, obtained from the spectrum of the transmitted pulse, are shown in Fig. 1 together with the spectrum of the incident pulse. We found bleaching of the exciton absorption without shifts of both the light-hole $(\mathrm{lh}-X)$ and the heavyhole (hh- $X$ ) exciton resonances as shown by Fig. 2 where the $\mathrm{hh}-X$ and $\mathrm{lh}-X$ peak position and peak absorption are dis-

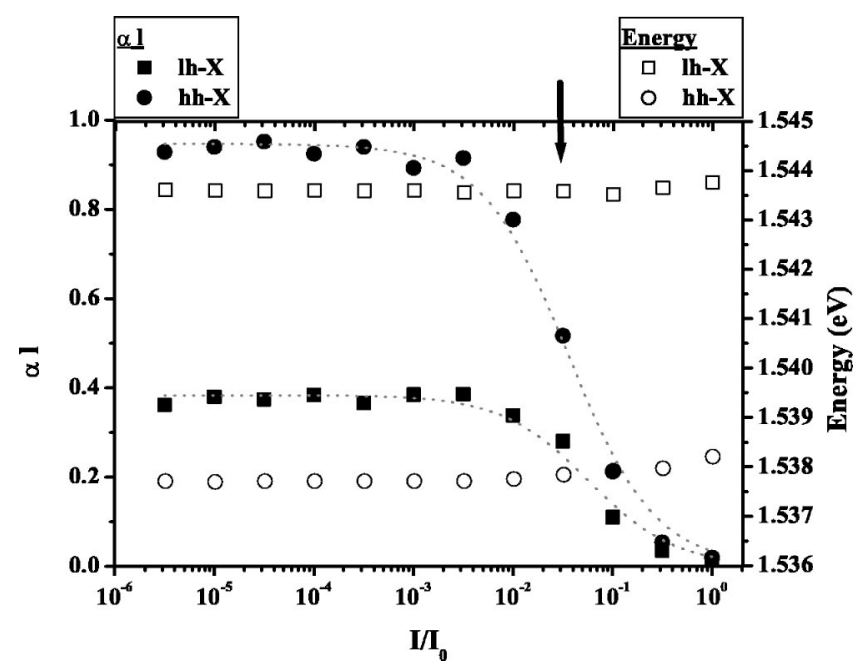

FIG. 2. Energy of the hh- $X$ and $\mathrm{lh}-X$ resonances (solid symbols) and corresponding peak absorption $\alpha l$ (open symbols) as a function of the normalized laser intensity $I / I_{0}$. The dotted line is a fit of the data to Eq. (12). The arrow indicates the intermediate intensity regime that we explore with the TI-FWM experiments with the incoherent background of carriers. 


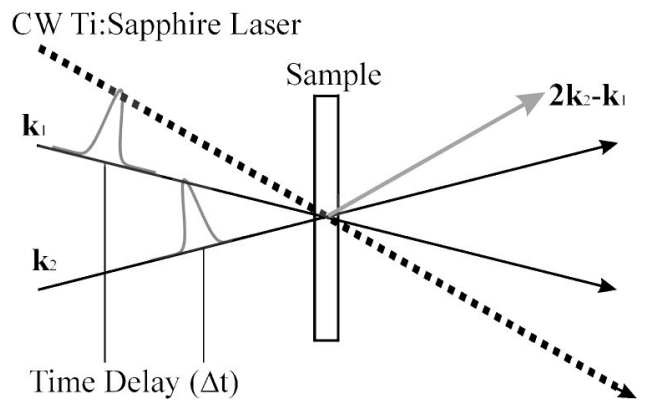

FIG. 3. Scheme of the four-wave mixing experimental setup. Two equal intensity pulses, with wave vectors $\mathbf{k}_{1}$ and $\mathbf{k}_{2}$, and a third cw laser beam are focused on the sample. The beams are linearly parallel polarized. The signal emitted in the $2 \mathbf{k}_{2}-\mathbf{k}_{1}$ direction is measured.

played. The absorption strength at both hh- $X$ and lh- $X$ peaks follows a simple saturation rule, ${ }^{28,29}$

$$
\alpha(I)=\alpha(0)\left(1+I / I_{\mathrm{s}}\right)^{-1},
$$

where $I$ is the laser intensity, $\alpha(0)$ is the linear absorption coefficient, $\alpha(I)$, the absorption at a laser intensity $I$, and $I_{\mathrm{s}}$ is the saturation intensity. We find that $I_{\mathrm{s}} \approx 300 \mathrm{~W} \mathrm{~cm}^{-2}$, in excellent agreement with early experiments. ${ }^{28,29}$ The constant energy of the two exciton resonances is caused by a redshift of the band gap which almost exactly cancels out the reduction of the exciton binding energy due to the increased screening the of electron-hole Coulomb attraction. ${ }^{30,31}$ This redshift is known as band-gap renormalization and is now theoretically well understood. ${ }^{32}$ For intermediate excitation intensity, an extra peak at the lower-energy side of hh- $X$ develops. It is ascribed to the formation of biexcitons $\left(X_{2}\right)$. A microscopic theory that quantitatively explains the power dependence of the transmission spectra has been provided by Jahnke and co-workers. ${ }^{33,34}$ Nevertheless it does not cover four-particle Coulomb correlation and, therefore, does not explain the biexcitonic peak. The biexciton binding energy $\left(E_{b}^{X_{2}}\right)$ deduced from the splitting between the hh- $X$ and biexciton, Fig. 1, is about $1.7 \mathrm{meV}$. This value is consistent with the findings of Birkedal, Singh, et al. on similar GaAs QW's. ${ }^{35,36}$ The biexciton energy can also be determined through the exciton-biexciton quantum beats seen in TIFWM as discussed below.

Next we perform TI-FWM on the sample without the background of incoherent carriers (Fig. 3). We used spectral shaping of the pulses to avoid exciting the $\mathrm{lh}-X$ and the continuum in order to observe clear biexcitonic effects. With the laser pulses shown in the inset of Fig. 4 the relative excitation of these species is such that $\mathcal{N}_{h h-X} \approx 25 \times \mathcal{N}_{l h-X} \gg \mathcal{N}_{c}$. The pulses in the $\mathbf{k}_{1}$ and $\mathbf{k}_{2}$ directions have approximately the same intensity and are linearly polarized with parallel polarization. We measured the TI-FWM signal in the $2 \mathbf{k}_{1}$ $-\mathbf{k}_{2}$ direction as a function of $\Delta t$, the time delay between the pulses, for many laser intensities on the sample creating $\mathcal{N}_{h h-X} \approx 1.6 \times 10^{9} \rightarrow 5 \times 10^{11} \mathrm{~cm}^{-2}$ hh- $X$ as shown in Fig. 4 . Several features of the TI-FWM signal are strongly affected by the laser intensity. Whereas at low excitation intensity the decay time, $T_{2}$, is constant, it clearly decreases strongly as

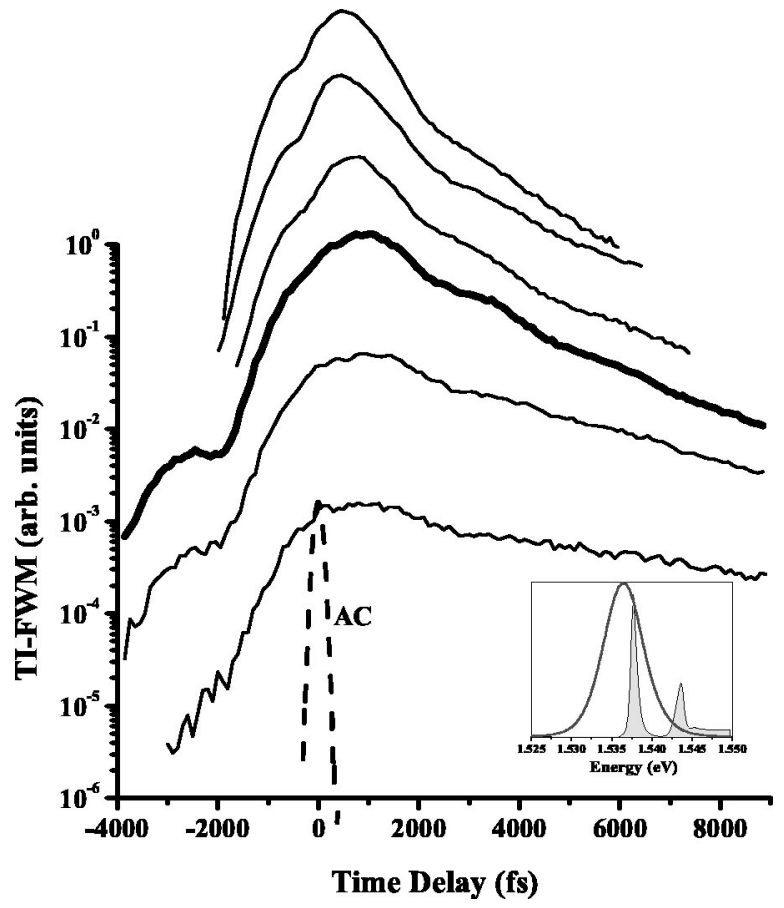

FIG. 4. TI-FWM signal without incoherent background of carriers. From top to bottom the excitation densities created at hh and lh, respectively, are $\mathcal{N} \equiv\left(\mathcal{N}_{l h-X}, \mathcal{N}_{h h-X}\right) \approx\left(0.19 \times 10^{11}, 5.0\right.$ $\left.\times 10^{11}\right) \mathrm{cm}^{-2}, \mathcal{N} \times 10^{-0.5}, \mathcal{N} \times 10^{-1.0}, \mathcal{N} \times 10^{-1.5}, \mathcal{N} \times 10^{-2.0}$, and $\mathcal{N} \times 10^{-2.5}$. A medium excitation (bold solid line) is used for the experiments with incoherent carriers (Fig. 5). The dashed line shows the laser-pulse autocorrelation. The laser spectrum overlaps mainly with the hh absorption line (inset).

that intensity increases, following roughly a power law, $1 / T_{2} \propto \mathcal{N}^{1 / 4}$. Although we took precautions to mostly excite the hh- $X$, at high excitation the admixture of excited species may change, therefore, we do not consider that the trends in the $T_{2}$ power dependence have a general significance. This point will be discussed in more detail in Sec. IV. More importantly, the data exhibits qualitative signatures of fourparticle correlation. The TI-FWM signal (i) increases very slowly for negative delays ${ }^{37}$ and (ii) exhibits beats for moderate excitation intensity. ${ }^{38}$ The beat frequency corresponds to a hh- $X$ biexciton splitting of about $2 \mathrm{meV}$ in good agreement with the findings of the transmission measurements. The optimal regime for observing a clear signature of fourparticle correlation is obtained for an excitation intensity $I=320 \mathrm{~W} \mathrm{~cm}^{-2}$ or a hh-X density $\mathcal{N}_{h h-X}=1.6$ $\times 10^{10} \mathrm{~cm}^{-2}$, corresponding to the bold curves in Figs. 1 and 4 .

We thus fixed the characteristics of the laser pulses onto the sample to the condition in which this TI-FWM profile is obtained and studied the changes induced by an incoherent background of carriers generated by the $\mathrm{cw}$ laser exciting the sample at $\hbar \omega_{\mathrm{cw}}=1.5694 \mathrm{eV}$. The density, $\mathcal{N}_{\mathrm{cw}}$, of carriers created by the $\mathrm{cw}$ laser is determined by the equilibrium condition $\mathcal{N}_{\mathrm{cw}} T_{1}^{-1}=g \alpha\left(\omega_{\mathrm{cw}}\right) l P_{\mathrm{cw}}$, where $g$ is a factor that contains the photon energy and the spot size. $P_{\mathrm{cw}}$ is the power of the $\mathrm{cw}$ laser and $T_{1}=100 \mathrm{ps}$ is the estimated electron-hole recombination time. It is known from time- 


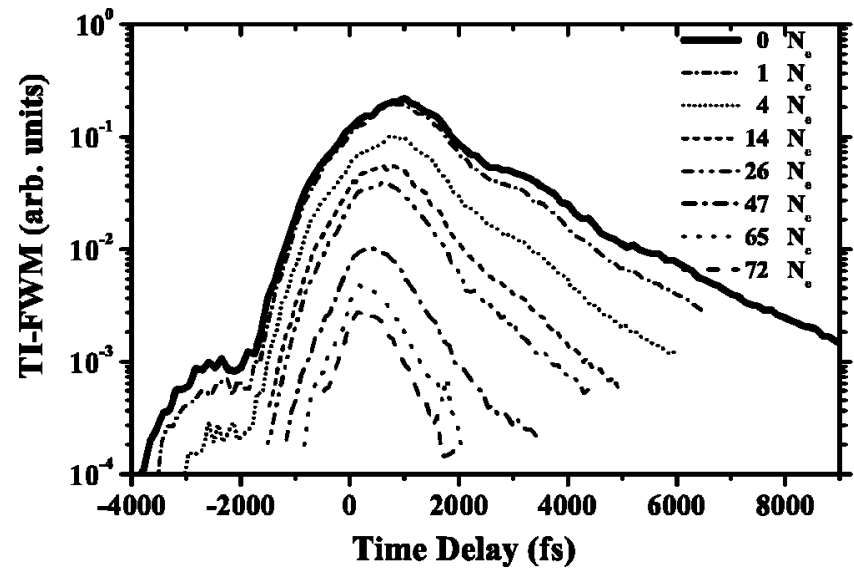

FIG. 5. TI-FWM signal for incoherent carrier densities $\mathcal{N}_{\mathrm{cw}}$ $=0 \rightarrow 5.8 \times 10^{11} \mathrm{~cm}^{-2}$ created by the cw laser at $1.5694 \mathrm{eV}(790$ $\mathrm{nm}$ ), where $\mathcal{N}_{\mathrm{e}}=8 \times 10^{9} \mathrm{~cm}^{-2}$ and the coherent carrier density is $1.6 \times 10^{10} \mathrm{~cm}^{-2}$.

resolved photoluminescence measurements that $T_{1}$ can be as short as 20 ps for cold resonantly excited carriers and as long as $1 \mathrm{~ns}$ for hot carriers. Figure 5 shows examples of the TI-FWM signal measured for $\mathcal{N}_{\mathrm{cw}}=0 \rightarrow 5.8 \times 10^{11} \mathrm{~cm}^{-2}$. The incoherent carriers produce very important changes in the TI-FWM signal: its overall duration and magnitude decrease strongly, and the four-particle correlation effects become less pronounced and eventually disappear at high excitation when the signal becomes extremely short. The decay time decreases by a factor of 2 following approximately the relation $T_{2}^{-1} \propto \mathcal{N}_{\mathrm{cw}}^{0.15}$ as shown by the solid symbols in Fig. 6 .

\section{COMPARISON AND DISCUSSION}

Considering the experimental excitation conditions, we can safely assume that only the lowest electronic subbands of the QW's are relevant. Since we are mostly interested in the

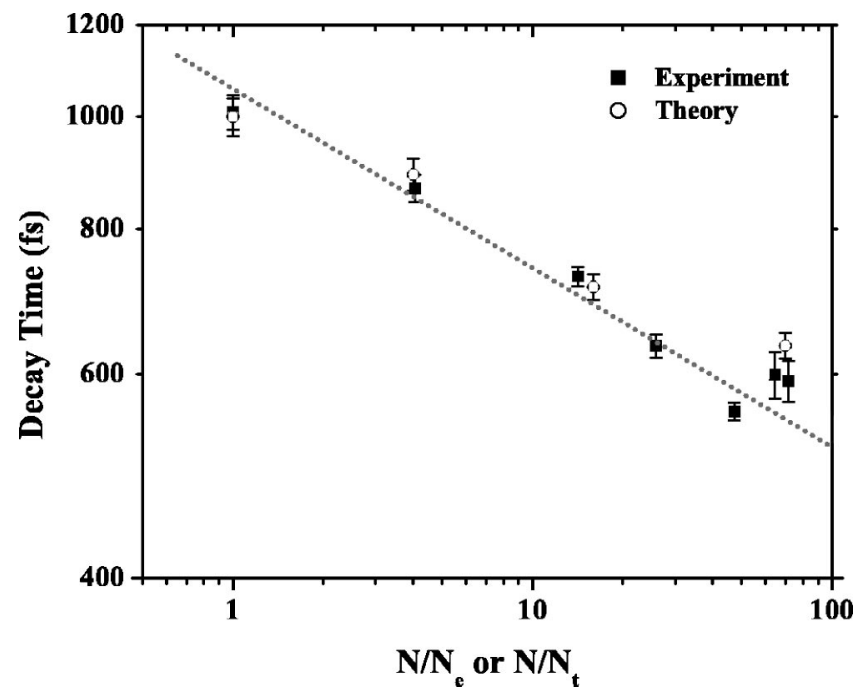

FIG. 6. Decay time $T_{2}$ of the TI-FWM signal for different densities $\mathcal{N}_{\mathrm{cw}}$ of incoherent background carriers. Solid squares are experimental, open circles theoretical results. The dashed line fits the decay times with $1 / T_{2} \propto \mathcal{N}^{0.15}$. many-body effects and not in predicting the precise exciton and biexciton energies, we assume infinitely high QW's. This simplifies the calculation of all matrix elements, while having only a minor influence on their magnitude as compared with finite height wells. Therefore, all the momentum variables in the equations of motion in Sec. II as well as in the following formulas must be understood as $2 \mathrm{D}$-quantities in the QW plane. The Coulomb interaction then is given by

$$
\begin{gathered}
w_{\mathbf{q}}=\frac{1}{\epsilon_{\mathbf{q}}} v_{\mathbf{q}}, \\
v_{\mathbf{q}}=\frac{e^{2}}{\epsilon_{0} \epsilon_{b} l}\left[\frac{3}{\mathbf{q}^{2} l^{2}+4 \pi^{2}}+\frac{8 \pi^{2}}{\mathbf{q}^{2} l^{2}\left(\mathbf{q}^{2} l^{2}+4 \pi^{2}\right)}\right. \\
\left.-\frac{32 \pi^{4}[1-\exp (-|\mathbf{q} l|)]}{|\mathbf{q} l|^{3}\left(\mathbf{q}^{2} l^{2}+4 \pi^{2}\right)^{2}}\right] .
\end{gathered}
$$

It is easy to verify that for large momenta the Coulomb potential behaves like in the 3D case, while for small momenta $v_{\mathbf{q}} \propto\left(1 / \mathbf{q} l+3 / 4 \pi^{2}\right)$ corresponds to the $2 \mathrm{D}$ case. Screening is accounted for by the dielectric function described by the Lindhardt formula:

$$
\epsilon_{\mathbf{q}}=1-v_{\mathbf{q}} \sum_{\mathbf{k}, a} \frac{f_{\mathbf{k}+\mathbf{q}}^{a}-f_{\mathbf{k}}^{a}}{\varepsilon_{\mathbf{k}+\mathbf{q}}^{a}-\varepsilon_{\mathbf{k}}^{a}}
$$

The one-particle distributions that enter Eq. (14) as well as Eqs. (8), (10), and (11) are obtained as solutions of their equations of motion. In general they are nonequilibrium distributions and incorporate the effects of the incoherent distributions created by the cw-laser as well as the contributions of the laser pulses that generate the FWM signals.

The equations of motion of the transition amplitude (2) and of the one-particle distributions are solved in momentum space. The equation of motion for the biexcitonic correlation (9) cannot be treated in momentum space. As usual we expand it in terms of excitonic eigenfunctions, which are solutions of the Wannier-like equation,

$$
\left[\varepsilon_{\mathbf{k}}^{e}+\varepsilon_{\mathbf{k}}^{h}\right] \varphi_{n, \mathbf{k}}^{e h}-\sum_{\mathbf{k}^{\prime}} w_{\mathbf{k}-\mathbf{k}^{\prime}} \varphi_{n, \mathbf{k}^{\prime}}^{e h}=\varepsilon_{n}^{e h} \varphi_{n, \mathbf{k}}^{e h}
$$

An important question that must be addressed is whether these eigenfunctions depend on screening. Since it is not possible to recalculate the matrix elements as screening builds up, we use the Coulomb potential at late times, when the distributions have reached a stationary state. This is a minor approximation, because the one-particle distributions build up with the excitation pulse and quickly become stationary.

In order to maintain the antisymmetry properties of the biexcitonic correlation function at all levels of the expansion, we write it as 


$$
\begin{aligned}
B_{\mathbf{k}+\mathbf{q}, \mathbf{k}, \mathbf{k}^{\prime}, \mathbf{k}^{\prime}+\mathbf{q}}^{e h e^{\prime} h^{\prime}}= & \frac{1}{2}\left[\bar{B}_{\mathbf{k}+\mathbf{q}, \mathbf{k}, \mathbf{k}^{\prime}, \mathbf{k}^{\prime}+\mathbf{q}}^{e h e^{\prime} h^{\prime}}-\bar{B}_{\mathbf{k}^{\prime}, \mathbf{k}, \mathbf{k}+\mathbf{q}, \mathbf{k}^{\prime}+\mathbf{q}}^{e^{\prime} h e h^{\prime}}\right. \\
& \left.-\bar{B}_{\mathbf{k}+\mathbf{q}, \mathbf{k}^{\prime}+\mathbf{q}, \mathbf{k}^{\prime}, \mathbf{k}}^{e h^{\prime} e^{\prime} h}+\bar{B}_{\mathbf{k}^{\prime}, \mathbf{k}^{\prime}+\mathbf{q}, \mathbf{k}+\mathbf{q}, \mathbf{k}}^{e^{\prime} h^{\prime} e}\right]
\end{aligned}
$$

Each of the new quantities follows the equation of motion (9) with only the first of the two source terms. The second term arises because of the antisymmetry of the biexcitonic relation. The new quantities can be expanded in terms of the eigenfunctions (15):

$$
\bar{B}_{\mathbf{k}+\mathbf{q}, \mathbf{k}, \mathbf{k}^{\prime}, \mathbf{k}^{\prime}+\mathbf{q}}^{e h e^{\prime} h^{\prime}}=\sum_{n m} B_{n m}^{e h e^{\prime} h^{\prime}}(\mathbf{q}) \varphi_{n, \mathbf{k}+\alpha \mathbf{q}}^{e h} \varphi_{m, \mathbf{k}^{\prime}+\beta \mathbf{q}}^{e^{\prime} h^{\prime}},
$$

where $\alpha=m_{h} /\left(m_{e}+m_{h}\right)$ and $\beta=m_{e} /\left(m_{e}+m_{h}\right)$. While this expansion maintains the antisymmetry of the biexcitonic correlation function, it does not separate singlet and triplet states. $^{18}$

The spectrum of the biexcitonic correlation function is obtained by solving the equation of motion in the frequency domain:

$$
\begin{aligned}
& {\left[\omega-\varepsilon_{n \mathbf{q}}^{e h}-\varepsilon_{m \mathbf{q}}^{e^{\prime} h^{\prime}}+i \hbar \gamma_{n m}\right] B_{n m}^{e h e^{\prime} h^{\prime}}(\mathbf{q} ; \omega)} \\
& \quad-\sum_{n^{\prime} m^{\prime} \mathbf{q}^{\prime}} w_{\mathbf{q}-\mathbf{q}^{\prime}} M_{n n^{\prime}}^{e h}\left(\mathbf{q}^{\prime}-\mathbf{q}\right) M_{m m^{\prime}}^{e^{\prime} h^{\prime}}\left(\mathbf{q}-\mathbf{q}^{\prime}\right) B_{n m}^{e h e^{\prime} h^{\prime}}\left(\mathbf{q}^{\prime}\right) \\
& \quad=\sum_{i j} w_{\mathbf{q}} M_{n i}^{e h}(\mathbf{q}) M_{m j}^{e^{\prime} h^{\prime}}(-\mathbf{q}),
\end{aligned}
$$

where the overlap matrix element $M_{n m}^{e h}(\mathbf{q})$ and the effective dephasing $\gamma_{n m}$ are defined in Appendix B. Since we are interested in the spectrum of the biexcitonic correlation only, the frequency dependence of the polarization is not included. After inverting Eq. (18) the spectrum is calculated by projecting $B_{n m}^{e h e^{\prime} h^{\prime}}(\mathbf{q} ; \omega)$ on the matrix element that couples the biexcitonic correlation to the polarization, defined in Appendix B:

$$
S(\omega)=\left|\sum_{n m \mathbf{q}} C_{n m}^{e h e^{\prime} h^{\prime}}(\mathbf{q}) B_{n m}^{e h e^{\prime} h^{\prime}}(\mathbf{q} ; \omega)\right|^{2} .
$$

Figure 7 shows the spectrum of the hh biexcitonic correlation function for different densities of incoherent carrier densities. For this calculation we have assumed that screening of the Coulomb interaction and dephasing is caused by a thermal distribution of free carriers. As the sample was held at a temperature of $5 \mathrm{~K}$, we assumed a carrier temperature of $10 \mathrm{~K}$ for this and all further calculations. We see that at low carrier densities the spectrum is dominated by the hh- $X_{2}$ bound biexciton. We note that for the parameters of our sample this calculation shows bound states of the hh- $X$ only, and not for the $1 \mathrm{~h}-X$ states or for $h \mathrm{~h}-X / \mathrm{lh}-X$ mixed states. As the carrier density increases the Coulomb interaction weakens due to screening and, therefore, the hh- $X_{2}$ binding energy decreases. At the same time its width increases as dephasing

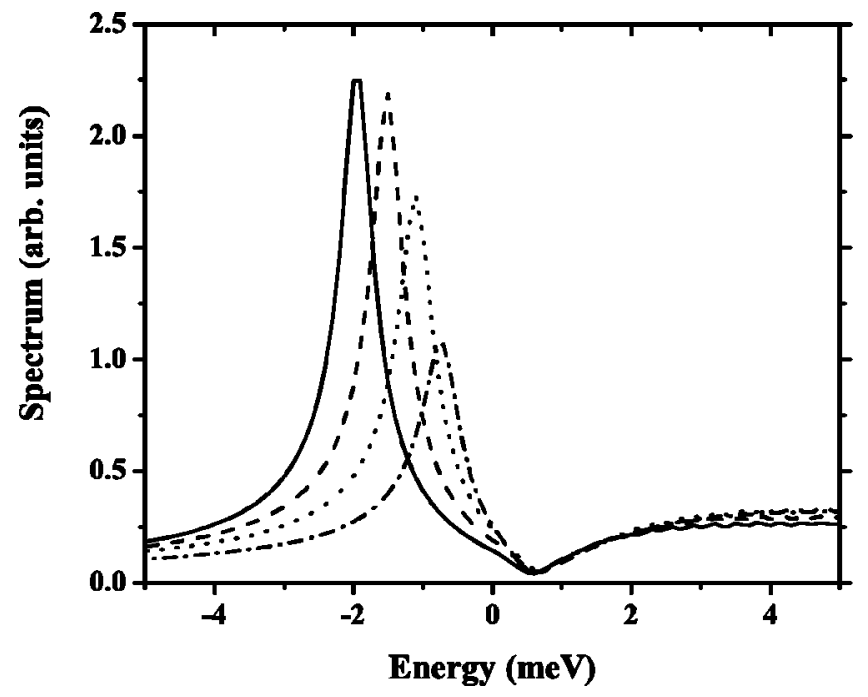

FIG. 7. Spectrum of the biexcitonic correlation function for incoherent carrier distributions of 0.45 (bold line), 0.9 (dashed), 1.75 (dotted) and $3.5 \times 10^{10} \mathrm{~cm}^{-2}$ (dot dashed). Energies are measured relative to twice the energy of the lowest exciton $2 \varepsilon_{0}^{e h}$.

gets stronger and the spectral weight decreases. At high densities finally the bound state disappears completely.

As shown experimentally, the FWM signal is influenced by both the incoherent carriers created by the cw laser and the carriers generated by the laser pulses that produce the FWM. Therefore, we need to separate the effects of these two species, especially in terms of their contributions to screening and dephasing. In the absence of incoherent carriers our starting point is experiments with a hh- $X$ density $\mathcal{N}_{h h-X}=1.6 \times 10^{10} \mathrm{~cm}^{-2}$ that correspond to the bold curves of Figs. 1 and 4, and we cannot simply assume that these carriers do not contribute to screening and dephasing. In order to account for them systematically, we calculate the absorption spectrum in the absence of incoherent carriers within the framework of our approach. Then we adjusted in our program the laser excitation density to reproduce the experimental hh- $X$ linewidth and the hh- $X_{2}$ binding energy. Finally, keeping the excitation density constant in the computation, we can calculate the TI-FWM signal for various densities of incoherent carriers as shown in Fig. 8.

The calculations are in good agreement with the experiments and reproduce the main features of the evolution of the TI-FWM signal as the incoherent carrier density is varied. As $\mathcal{N}_{\mathrm{cw}}$ increases both the rise time, for $\Delta t<0$, and the decay time, for $\Delta t>0$, decrease quickly. The theory accounts quantitatively for the observed overall decrease of the signal strength and the shift of its temporal maximum, which at low densities moves towards positive $\Delta t$. This later effect is due to the influence of biexcitonic correlations. When $\mathcal{N}_{\mathrm{cw}}$ increases the biexcitonic correlation weakens, because of the more effective screening and of dephasing by scattering with these carriers. The FWM signal therefore approaches the limit of semiconductor Bloch equation (SBE) with, in particular, a maximum at $\Delta t=0$. The decreasing contribution of biexcitonic correlations is seen most clearly on the $\Delta t<0$ signal where oscillations, corresponding to $\mathrm{hh}-X_{2}$ binding 


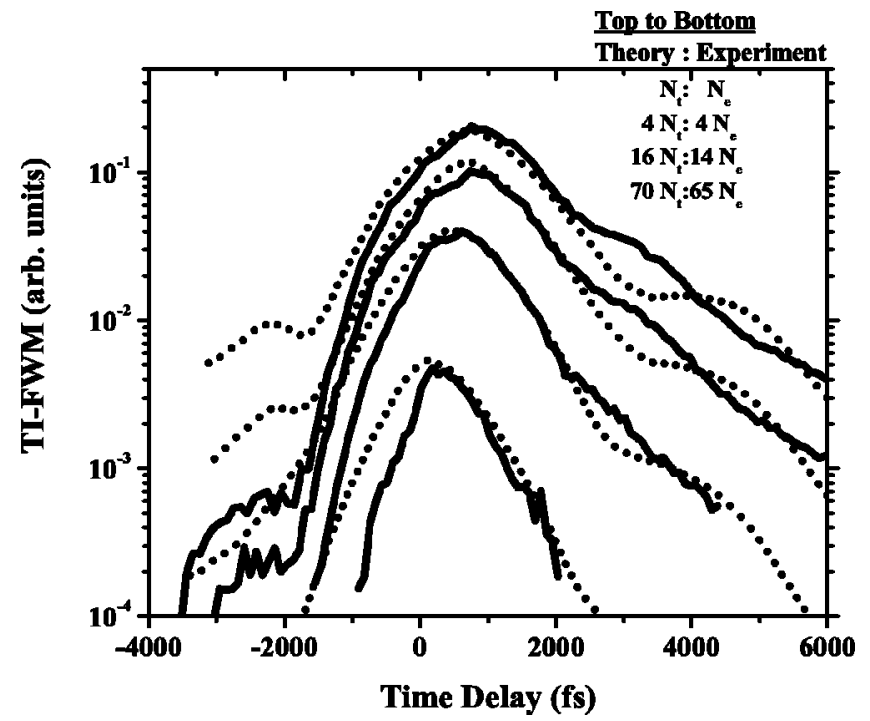

FIG. 8. TI-FWM spectra for different incoherent carrier densities, where $\mathcal{N}_{\mathrm{t}}=8.8 \times 10^{9} \mathrm{~cm}^{-2}$ and $\mathcal{N}_{\mathrm{e}} \approx 8 \times 10^{9} \mathrm{~cm}^{-2}$. The solid lines are experimental data while dashed lines are theoretical simulation results. We attribute the difference between $\mathcal{N}_{\mathrm{e}}$ and $\mathcal{N}_{\mathrm{t}}$ to the uncertainty in the recombination lifetime.

energy, disappear as screening and dephasing increase. The difference in the beating periods of experiment and theory is attributed to slightly different biexciton binding energies for experiment and theory, which in turn is due to the assumption of infinitely high quantum wells. Also, the SBE predicts a fixed ratio of 2 between the TI-FWM signal decay time and rise time. This is seen in the high incoherent carrier density calculation, but for low densities the rise time is significantly longer, again because of biexcitonic correlations. In other systems, such as magnetoexcitons in bulk GaAs, this rise time can become much longer than the decay time. ${ }^{39,18} \mathrm{Ex}-$ perimentally we found that, in the range of density explored here, the decay time decreases by a factor of 2 following approximately the relation $T_{2}^{-1} \propto \mathcal{N}_{\mathrm{cw}}^{0.15}$, see the solid symbols in Fig. 6. The open symbols in that figure are the theoretical results again in excellent agreement with the data. We note, however, that the experimental FWM signal rise time is significantly smaller than calculated. We attribute the differences to an inhomogenous broadening that is not accounted for in the calculation.

At this point it is worth discussing the general issue of the density dependence of dephasing. It has been advanced that the dephasing rate, $\gamma=1 / T_{2}$, should follow a power law: $\gamma$ $\propto \mathcal{N}^{1 / 3}$ in three dimension and $\gamma \propto \mathcal{N}^{1 / 2}$ in two dimensions. ${ }^{40,41}$ The origin of this dependence can be traced back to the scaling of the wave-vector dependence of the one-particle distribution functions and the Coulomb interaction. ${ }^{42}$ Ultimately it is wave-vector dependence of the carrier-carrier scattering rate, which translates into an energy dependence in optical spectra, and determines also the density dependence. These theoretical considerations, however, assume a thermal carrier distribution. Other dependencies have been discussed for the $2 \mathrm{D}$ case as well. ${ }^{43}$ Generally, dephasing should strongly depend on the actual $k$-space distribution. Since in most experiments it is strongly nonequi-

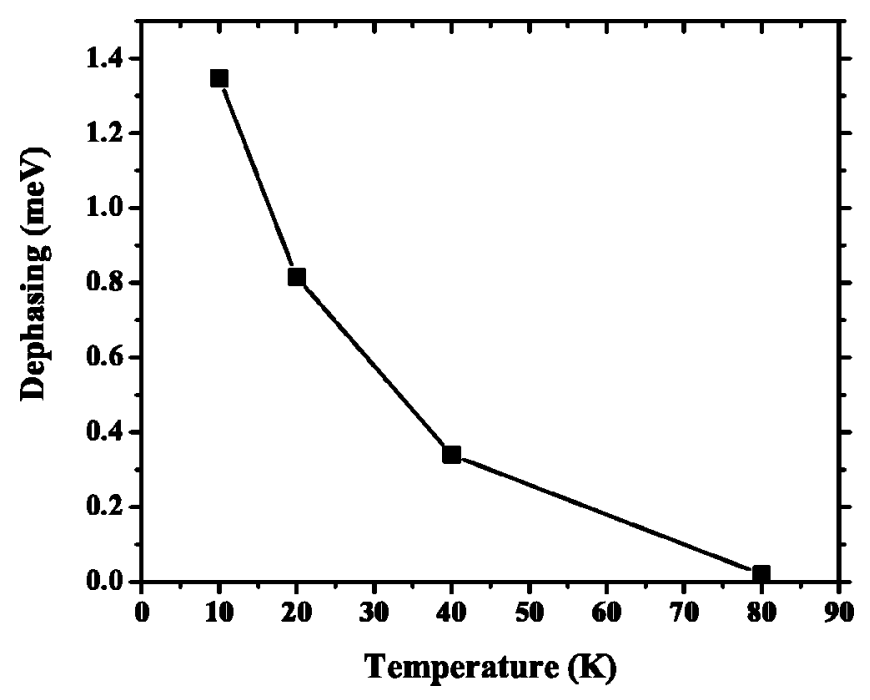

FIG. 9. Temperature dependence of the dephasing of the lowest $h h$ exciton for a carrier density of $2 \times 10^{11} \mathrm{~cm}^{2}$.

librium, these power laws cannot be valid under all conditions. To explore this effect we calculated the dephasing rate using Eq. (8) for different incoherent carrier temperatures. In order to eliminate the $k$ dependence and get an experimentally accessible result, we projected Eq. (8) on the lowest exciton level, thus obtaining $\gamma_{h h-X}$ for the broadening of that level only. As shown in Fig. 9 we find that $\gamma_{h h-X}$ varies strongly with the carrier temperature. With increasing temperature and therefore for broader carrier distribution in $k$ space, the dephasing gets weaker. It should be remembered that initially the nonequilibrium distributions created by laser pulses (in particular in our experiments the pulses that produce the FWM in absence of the cw excitation) are narrow in $k$ space. Thus, for the same amount of carriers, the dephasing induced by pulsed lasers is stronger than that of thermal distributions. Furthermore, these nonequilibrium distributions depend on the details of the sample structure and, therefore, we believe it is impossible to derive a general power law for $\gamma_{h h-X}$.

\section{CONCLUSION}

In conclusion we have demonstrated that our theoretical approach is able to describe the microscopic dynamics of laser-excited semiconductors quantitatively for all excitation densities at the $\chi^{(3)}$ level. This is achieved by accounting on the same footing for two important effects: (i) scattering with free carriers as the most effective dephasing process and (ii) screening of the Coulomb interaction that controls correlation effects. The calculations shown here can easily be improved on several points, for example, by accounting for the QW finite height or including inhomogeneous broadening. This would require more computation but no conceptual advance. However, the issue of extending our treatment to all order in the applied optical field is still open.

\section{ACKNOWLEDGMENTS}

We would like to thank the Sandia National Laboratory for providing us with high-quality samples. The work at Ber- 
keley was supported by the Director, Office of Science, Office of Basic Energy Science, Division of Materials Sciences and Office of Science, U. S. Department of Energy under Contract No. DE-AC03-76SF00098. R. L. and D. H. gratefully acknowledge support by the Alexander von Humboldt Foundation.

\section{APPENDIX A: DERIVATION}

The derivation of our approach is done by a diagrammatic analysis of the scattering terms in Eq. (2). Our starting point is the second-order Born approximation in valence- and conduction-band notation:

$$
\begin{aligned}
\left.\frac{\partial}{\partial t} P_{\mathbf{k}}^{c v}(t)\right|_{\text {scatt }}= & \left.i \hbar \frac{\partial}{\partial t} G_{c v \mathbf{k}}^{<}(t, t)\right|_{\text {scatt }} \\
= & i \hbar \sum_{a a^{\prime} b=\{c, v\}} \sum_{\mathbf{k}^{\prime} \mathbf{q}} \int_{-\infty}^{t} d t^{\prime}\left\{\left[w _ { \mathbf { q } } ^ { 2 } \left(G_{c a \mathbf{k}+\mathbf{q}}^{>}\left(t, t^{\prime}\right) G_{a^{\prime} b \mathbf{k}^{\prime}+\mathbf{q}^{\prime}}^{<}\left(t^{\prime}, t\right) G_{b a^{\prime} \mathbf{k}^{\prime}}^{>}\left(t, t^{\prime}\right) G_{a v \mathbf{k}}^{<}\left(t^{\prime}, t\right)-G_{c a \mathbf{k}}^{>}\left(t, t^{\prime}\right)\right.\right.\right. \\
& \left.\times G_{a b \mathbf{k}+\mathbf{q}}^{<}\left(t^{\prime}, t\right) G_{a^{\prime} b \mathbf{k}^{\prime}+\mathbf{q}}^{>}\left(t, t^{\prime}\right) G_{a^{\prime} v \mathbf{k}^{\prime}}^{<}\left(t^{\prime}, t\right)\right)-w_{\mathbf{q}} w_{\mathbf{k}-\mathbf{k}^{\prime}}\left(G_{c a^{\prime} \mathbf{k}+\mathbf{q}}^{>}\left(t, t^{\prime}\right) G_{a^{\prime} b \mathbf{k}^{\prime}+\mathbf{q}}^{<}\left(t^{\prime}, t\right)\right. \\
& \left.\left.\left.\times G_{b a^{\prime} \mathbf{k}^{\prime}}^{>}\left(t, t^{\prime}\right) G_{a v \mathbf{k}}^{<}\left(t^{\prime}, t\right)-G_{c a \mathbf{k}}^{>}\left(t, t^{\prime}\right) G_{a a^{\prime} \mathbf{k}+\mathbf{q}^{\prime}}^{<}\left(t^{\prime}, t\right) G_{a^{\prime} b \mathbf{k}^{\prime}+\mathbf{q}^{\prime}}^{>}\left(t, t^{\prime}\right) G_{b v \mathbf{k}^{\prime}}^{<}\left(t^{\prime}, t\right)\right)\right]+\left[G^{<} \rightleftharpoons G^{>}()\right]\right\} .
\end{aligned}
$$

First we want to motivate our ansatz to reduce the twotime Green's functions to single-time functions. For statically interacting quasiparticles the Green's function satisfies the Dyson equation

$$
\begin{aligned}
G_{c v}^{<}\left(t, t^{\prime}\right)= & \int_{-\infty}^{t} d t^{\prime \prime} \sum_{n} G_{c c}^{0 r}\left(t, t^{\prime \prime}\right) \Sigma_{c n}^{r}\left(t^{\prime \prime}\right) G_{n v}^{<}\left(t^{\prime \prime}, t^{\prime}\right) \\
& +\int_{-\infty}^{t} d t^{\prime \prime} \sum_{n} G_{c c}^{0 r}\left(t, t^{\prime \prime}\right) \Sigma_{c n}^{<}\left(t^{\prime \prime}\right) G_{n v}^{a}\left(t^{\prime \prime}, t^{\prime}\right),
\end{aligned}
$$

where $\Sigma_{c v}^{r}()=\Omega_{c v}$ is the renormalized Rabi energy and $\Sigma_{c c}^{r}()=\Sigma^{\text {st }}$ the static, Hartree-Fock contribution to the oneparticle energy. We suppress the momentum variable for the meantime. In the low-density limit electron and hole distributions are small, so that we have $-i \hbar G_{v v}^{<}()=i \hbar G_{c c}^{>}() \approx 1$ and $G_{v v}^{>}()=G_{c c}^{<}() \approx 0$. Under this condition only the first term in Eq. (A2) contributes and $G_{c v}^{<}\left(t, t^{\prime}\right)$ depends indeed on the first time argument only. Similarly $G_{v c}^{<}\left(t, t^{\prime}\right)$ depends on the second argument only. These considerations lead us to the ansatz

$$
G_{c v \mathbf{k}}^{<}\left(t, t^{\prime}\right)=i \hbar \sum_{n} G_{c n \mathbf{k}}^{<}(t, t) \times\left[G_{n v \mathbf{k}}^{r}\left(t, t^{\prime}\right)-G_{n v \mathbf{k}}^{a}\left(t, t^{\prime}\right)\right]
$$

$$
G_{v c \mathbf{k}}^{>}\left(t, t^{\prime}\right)=i \hbar \sum_{n} G_{v n \mathbf{k}}^{>}(t, t) \times\left[G_{n c \mathbf{k}}^{r}\left(t, t^{\prime}\right)-G_{n c \mathbf{k}}^{a}\left(t, t^{\prime}\right)\right],
$$

$$
G_{c v \mathbf{k}}^{>}\left(t, t^{\prime}\right)=i \hbar \sum_{n}\left[G_{c n \mathbf{k}}^{r}\left(t, t^{\prime}\right)-G_{c n \mathbf{k}}^{a}\left(t, t^{\prime}\right)\right] \times G_{n v \mathbf{k}}^{>}\left(t^{\prime}, t^{\prime}\right),
$$

and

$$
G_{v c \mathbf{k}}^{<}\left(t, t^{\prime}\right)=i \hbar \sum_{n}\left[G_{v n \mathbf{k}}^{r}\left(t, t^{\prime}\right)-G_{v n \mathbf{k}}^{a}\left(t, t^{\prime}\right)\right] \times G_{n c \mathbf{k}}^{<}\left(t^{\prime}, t^{\prime}\right) .
$$

These relations reproduce the low-density limit known from DCTS correctly.

For simplicity we assume a statically screened Coulomb interaction $w_{\mathbf{q}}$. The contributions $\propto w_{\mathbf{q}}^{2}$ describe the random-

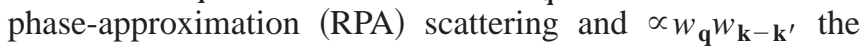
exchange terms. We avoid writing Eq. (A1) as products of self-energies and Green's functions because they will become coupled by the Coulomb interaction for higher-order contributions, as we will see soon.

In order to recover the contribution from coherent biexcitonic correlations known from DCTS, we have to couple excitonic polarizations by Coulomb interaction up to infinite order. In second order these interaction processes are usually referred to as polarization scattering. One RPA diagram is shown in the upper part of Fig. 10. There are corresponding diagrams, for which the RPA bubble is not given by the product of two polarizations but of two diagonal elements. As mentioned the coherent part of these one-particle distribution functions is determined by the product of two polarization functions according to Eq. (4). These contributions have to be renormalized in the same way as the RPA and exchange contributions of the polarization scattering. Assuming that electron and hole distributions are small compared to unity, we can neglect the terms indicated by $\left[G^{<}() \rightleftharpoons G^{>}()\right]$ in Eq. (A1).

Inserting Coulomb interactions for the third order for the upper diagram in Fig. 10 leads to the lower diagrams, where we have suppressed the momentum vectors. These diagrams represent simply the six possible interactions between two electrons and two holes and constitute the basic unit of interaction for biexcitonic correlations. In the same way we can now introduce fourth-order Coulomb interactions for 

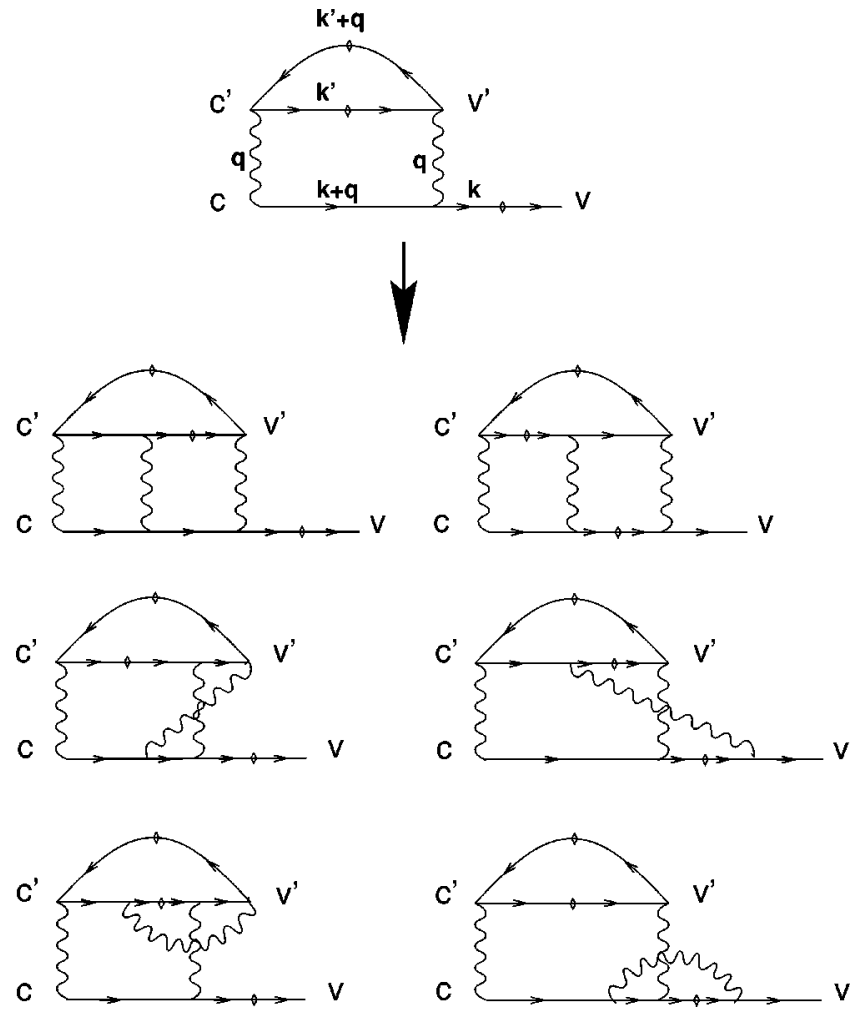

FIG. 10. RPA scattering contribution in second-order Born approximation and the third-order Coulomb renormalization which leads to biexcitonic correlations. The wavy lines represent Coulomb interactions, the solid arrows the diagonal elements of the Green's functions, and the lines with diamonds the off-diagonal elements.

each of these diagrams. The only restriction to the interaction lines is that those connecting a fixed pair of particles need to be ladderlike.

With the low-density ansatz Eqs. (5) and (6) we are able to evaluate those diagrams. We can write the $n$ th-order scattering contribution leading to biexcitonic contributions as

$$
\left.\frac{\partial}{\partial t} P_{\mathbf{k}}^{e h(n)}(t)\right|_{B}=\sum_{\mathbf{k}^{\prime} \mathbf{q}, e^{\prime} h^{\prime}} w_{\mathbf{q}} P_{\mathbf{k}^{\prime}}^{h^{\prime} e^{\prime}}(t) B_{\mathbf{k}+\mathbf{q}, \mathbf{k}, \mathbf{k}^{\prime}, \mathbf{k}^{\prime}+\mathbf{q}}^{e h h^{\prime} h^{\prime}(n-1)} .
$$

Diagrams like the upper part of Fig. 10 together with the corresponding coherent contribution of the diagonal Green's functions give the first-order biexcitonic correlation:

$$
\begin{aligned}
B_{\mathbf{k}+\mathbf{q}, \mathbf{k}, \mathbf{k}^{\prime}, \mathbf{k}^{\prime}+\mathbf{q}}^{e h h^{\prime}}(t)= & w_{\mathbf{q}} \int_{-\infty}^{t} d t^{\prime} \exp \left[-i\left(\varepsilon_{\mathbf{k}+\mathbf{q}}^{e}+\varepsilon_{\mathbf{k}}^{h}+\varepsilon_{\mathbf{k}^{\prime}}^{e^{\prime}}\right.\right. \\
& \left.\left.+\varepsilon_{\mathbf{k}^{\prime}+\mathbf{q}}^{h^{\prime}}\right)\left(t-t^{\prime}\right)\right] \times\left[P_{\mathbf{k}}^{e h}\left(t^{\prime}\right)-P_{\mathbf{k}+\mathbf{q}^{\prime}}^{e h}\left(t^{\prime}\right)\right] \\
& \times\left[P_{\mathbf{k}^{\prime}}^{e^{\prime} h^{\prime}}\left(t^{\prime}\right)-P_{\left.\left.\mathbf{k}^{\prime}+\mathbf{q}^{\prime} h^{\prime}\right)\right] .}^{e^{\prime}}\left(t^{\prime}\right)\right.
\end{aligned}
$$

The second-order correlation function is given by

$$
\begin{aligned}
& B_{\mathbf{k}+\mathbf{q}, \mathbf{k}, \mathbf{k}^{\prime}, \mathbf{k}^{\prime}+\mathbf{q}^{e h}}^{e h h^{\prime}}(t)=\sum_{\mathbf{q}^{\prime}} w_{\mathbf{q}^{\prime}} \int_{-\infty}^{t} d t^{\prime} \exp \left[-i\left(\varepsilon_{\mathbf{k}+\mathbf{q}}^{e}+\varepsilon_{\mathbf{k}}^{h}\right.\right. \\
& \left.\left.+\varepsilon_{\mathbf{k}^{\prime}}^{e^{\prime}}+\varepsilon_{\mathbf{k}^{\prime}+\mathbf{q}}^{h^{\prime}}\right)\left(t-t^{\prime}\right)\right] \\
& \times\left[B_{\mathbf{k}+\mathbf{q}^{+}+\mathbf{q}^{\prime}, \mathbf{k}, \mathbf{k}^{\prime}-\mathbf{q}^{\prime}, \mathbf{k}^{\prime}+\mathbf{q}^{\prime}}^{\left.e t^{\prime}\right)}\right. \\
& +B_{\mathbf{k}+\mathbf{q}, \mathbf{k}+\mathbf{q}^{\prime}, \mathbf{k}^{\prime}, \mathbf{k}^{\prime}+\mathbf{q}^{+}+\mathbf{q}^{\prime}}^{e h h^{\prime}}\left(t^{\prime}\right) \\
& -B_{\mathbf{k}+\mathbf{q}^{+}+\mathbf{q}^{\prime}, \mathbf{k}+\mathbf{q}^{\prime}, \mathbf{k}^{\prime}, \mathbf{k}^{\prime}+\mathbf{q}^{\prime}}^{\left.e h t^{\prime}\right)} \\
& -B_{\mathbf{k}+\mathbf{q}, \mathbf{k}, \mathbf{k}^{\prime}+\mathbf{q}^{\prime}, \mathbf{k}^{\prime}+\mathbf{q}^{+}+\mathbf{q}^{\prime}}\left(t^{\prime}\right) \\
& -B_{\mathbf{k}+\mathbf{q}^{+} \mathbf{q}^{\prime}, \mathbf{k}, \mathbf{k}^{\prime}, \mathbf{k}^{\prime}+\mathbf{q}^{+}+\mathbf{q}^{\prime}}\left(t^{\prime}\right)
\end{aligned}
$$

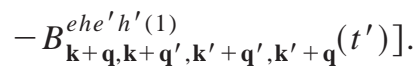

Higher order contributions to the biexcitonic correlation function lead to recursion formulas like Eq. (A9), due to the ladder approximation that we imposed on the Coulomb renormalization. The exchange contributions to Eq. (A8) are calculated in the same way. Summing up all orders we can map the resulting integral equation onto the equation of motion (9), except for the self-energy terms.

\section{APPENDIX B: MATRIX ELEMENTS}

The overlap matrix element is defined as

$$
M_{i n}^{e h}(\mathbf{q})=\sum_{\mathbf{k}} \varphi_{i, k}^{e h}\left[\varphi_{n, \mathbf{k}-\alpha \mathbf{q}}^{e h}-\varphi_{n, \mathbf{k}+\beta \mathbf{q}}^{e h}\right]
$$

The effective dephasing of the biexcitonic correlation function is given by

$$
\begin{gathered}
\hbar \gamma_{n m}=\hbar \gamma_{B}+\sigma_{n}^{e h}+\sigma_{m}^{e^{\prime} h^{\prime}}, \\
\sigma_{n}^{e h}=\sum_{\mathbf{k q}} \varphi_{n, \mathbf{k}+\alpha \mathbf{q}}^{e h 2}\left[\sigma_{\mathbf{k}+\mathbf{q}}^{e}+\sigma_{\mathbf{k}}^{h}\right] \\
+\sum_{\mathbf{k q q ^ { \prime }}} \varphi_{n, \mathbf{k}+\alpha \mathbf{q}}^{e h 2}\left[\sigma_{\mathbf{k}+\mathbf{q}, \mathbf{q}^{\prime}}^{e}+\sigma_{\mathbf{k}, \mathbf{q}^{\prime}}^{h}\right] .
\end{gathered}
$$

In the actual calculations $\gamma_{B}$ was set to 0 .

The matrix element coupling the biexcitonic correlation to the polarization is defined as

$$
\begin{aligned}
C_{n m}^{e h e^{\prime} h^{\prime}}(\mathbf{q})= & -w_{\mathbf{q}} M_{0 n}^{e h}(-\mathbf{q}) M_{0 m}^{e^{\prime} h^{\prime}}(\mathbf{q}) \\
& +\sum_{\mathbf{k} \mathbf{k}^{\prime}} w_{\mathbf{k}-\mathbf{k}^{\prime}} \varphi_{n, \mathbf{k}+\alpha \mathbf{q}}^{e h} \varphi_{m, \mathbf{k}+\beta \mathbf{q}}^{e^{\prime} h^{\prime}}\left[\varphi_{0, \mathbf{k}}^{e h}-\varphi_{0, \mathbf{k}^{\prime}}^{e h}\right] \\
& \times\left[\varphi_{0, \mathbf{k}^{\prime}+\mathbf{q}}^{e^{\prime} h^{\prime}}-\varphi_{0, \mathbf{k}+\mathbf{q}}^{e^{\prime} h^{\prime}}\right] .
\end{aligned}
$$


${ }^{1}$ D.S. Chemla, Ultrafast Transient Nonlinear Optical Processes in Semiconductors, edited by R. K. Willardson and A. C. Beers (Academic, New York, 1999).

${ }^{2}$ D.S. Chemla and J. Shah, Nature (London) 411(6837), 549 (2001).

${ }^{3}$ K. Leo, M. Wegener, J. Shah, D.S. Chemla, E.O. Göbel, T.C. Damen, and S. Schmitt-Rink, Phys. Rev. Lett. 65(11), 1340 (1990).

${ }^{4}$ M. Wegener, D.S. Chemla, S. Schmitt-Rink, and W. Schäfer, Phys. Rev. A 42(9), 5675 (1990).

${ }^{5}$ M.-A. Mycek, S. Weiss, J.-Y. Bigot, S. Schmitt-Rink, D.S. Chemla, and W. Schaefer, Appl. Phys. Lett. 60(21), 2666 (1992).

${ }^{6}$ S. Weiss, M.-A. Mycek, J.-Y. Bigot, S. Schmitt-Rink, and D.S. Chemla, Phys. Rev. Lett. 69(18), 2685 (1992).

${ }^{7}$ D.-S. Kim, J. Shah, T. Damen, W. Schäfer, F. Jahnke, S. SchmittRink, and K. Köhler, Phys. Rev. Lett. 69(18), 2725 (1992).

${ }^{8}$ S. Bar-Ad, P. Kner, M.V. Marquezini, D.S. Chemla,and K. El Sayed, Phys. Rev. Lett. 77(15), 3177 (1996).

${ }^{9}$ F.X. Camescasse, A. Alexandrou, D. Hulin, L. Bnyai, D.B. Tran Thoai, and H. Haug, Phys. Rev. Lett. 77(27), 5429 (1996).

${ }^{10}$ M.U. Wehner, D.S. Chemla, and M. Wegener, Phys. Rev. B 58(7), 3590 (1998).

${ }^{11}$ M.U. Wehner, M.H. Ulm, D.S. Chemla, and M. Wegener, Phys. Rev. Lett. 80(9), 1992 (1998).

${ }^{12}$ D. Steinbach, W. Hügel, and M. Wegener, J. Opt. Soc. Am. B 15(3), 1231 (1998).

${ }^{13}$ W.A. Hügel, M.F. Heinrich, Q.T. Wegener, M. Vu, L. Bányai, and H. Haug, Phys. Rev. Lett. 83(16), 3313 (1999).

${ }^{14}$ M. Wegener and D.S. Chemla, Chem. Phys. 251(1-3), 269 (2000).

${ }^{15}$ V.M. Axt and A. Stahl, Z. Phys. B: Condens. Matter 93, 195 (1994)

${ }^{16}$ K. Victor, V.M. Axt, and A. Stahl, Phys. Rev. B 51(20), 14164 (1995).

${ }^{17}$ W. Schäfer, D.S. Kim, J. Shah, T.C. Damen, J.E. Cunningham, K.W. Goossen, L.N. Pfeiffer, and K. Köhler, Phys. Rev. B 53(24), 16429 (1996).

${ }^{18}$ W. Schäfer, R. Lövenich, N.A. Fromer, and D.S. Chemla, Phys. Status Solidi B 221, 195 (2000).

${ }^{19}$ M.Z. Maialle and L.J. Sham, Phys. Rev. Lett. 73(24), 3310 (1994).

${ }^{20}$ N.H. Kwong and R. Binder, Phys. Rev. B 61(12), 8341 (2000).

${ }^{21}$ W. Schäfer, J. Opt. Soc. Am. B 13(6), 1291 (1996).

${ }^{22}$ L. Bányai, Q.T. Vu, B. Mieck, and H. Haug, Phys. Rev. Lett. 81, 882 (1998).
${ }^{23}$ M. Bonitz, D. Semkat, N. H. Kwong, and D. Kremp, in Proceedings of the Conference on Strongly Coupled Coulomb Systems, edited by G. Kalmann (Plenum, New York, 1998).

${ }^{24}$ W. Schäfer, R. Lövenich, N.A. Fromer, and D.S. Chemla, Phys. Rev. Lett. 86(2), 344 (2001).

${ }^{25}$ L. Schultheis, J. Kuhl, A. Honold, and C.W. Tu, Phys. Rev. Lett. 57, 1635 (1986).

${ }^{26}$ Quantum Theory of the Optical and Electronic Properties of Semiconductors, edited by H. Haug and S.W. Koch, 3rd ed. (World Scientific, Singapore, 1994).

${ }^{27}$ P. Lipavský, V. Spicka, and B. Velický, Phys. Rev. B 34, 6933 (1986).

${ }^{28}$ D.A.B. Miller, D.S. Chemla, D.J. Eilenberger, P.W. Smith, A.C. Gossard, and W.T. Tsang, Appl. Phys. Lett. 41, 679 (1982).

${ }^{29}$ D.S. Chemla, D.A.B. Miller, P.W. Smith, A.C. Gossard, and W. Wiegmann, IEEE J. Quantum Electron. 20, 265 (1984).

${ }^{30}$ H. Haug and S. Schmitt-Rink, Prog. Quantum Electron. 9, 3 (1985).

${ }^{31}$ S. Schmitt-Rink and C. Ell, J. Lumin. 30, 585 (1985).

${ }^{32}$ G. Tränkle, H. Leier, A. Forchel, H. Haug, C. Ell, and G. Weimann, Phys. Rev. Lett. 58(4), 419 (1987).

${ }^{33}$ F. Jahnke, M. Kira, S.W. Koch, G. Khitrova, E. Lindmark, T. Nelson, D. Wick, O. Lyngnes, H. Gibbs, and K. Tai, Phys. Rev. Lett. 77, 5257 (1996).

${ }^{34}$ F. Jahnke, M. Kira, and S.W. Koch, Z. Phys. B: Condens. Matter 104(3), 559 (1997).

${ }^{35}$ D. Birkedal, J. Singh, V.G. Lyssenko, J. Erland, and J.M. Hvam, Phys. Rev. Lett. 76(4), 672 (1996).

${ }^{36}$ I.K. Oh and J. Singh, Phys. Rev. B 60(4), 2528 (1999).

${ }^{37}$ P. Kner, W. Schäfer, R. Lövenich, and D.S. Chemla, Phys. Rev. Lett. 81(24), 5386 (1998).

${ }^{38}$ E.J. Mayer, G.O. Smith, V. Heuckeroth, J. Kuhl, K. Bott, A. Schulze, T. Meier, S.W. Koch, P. Thomas, R. Hey and K. Ploog, Phys. Rev. B 51(16), 10909 (1995).

${ }^{39}$ P. Kner, S. Bar-Ad, M. Marquezini, D.S. Chemla, R. Lövenich, and W. Schäfer, Phys. Rev. B 60(7), 4731 (1999).

${ }^{40}$ J.-Y. Bigot, M.T. Portella, R.W. Schoenlein, J.E. Cunningham, and C.V. Shank, Phys. Rev. Lett. 97(5), 636 (1991).

${ }^{41}$ D.W. Snoke, Phys. Rev. B 50, 11583 (1994).

${ }^{42}$ W. Schäfer and M. Wegener, Semiconductor Optics and Transport Phenomena, Advanced Texts in Physics (Springer, Berlin, 2002).

${ }^{43}$ B. Mieck, H. Haug, W.A. Hügel, M.F. Heinrich, and M. Wegener, Phys. Rev. B 62, 2686 (2000). 